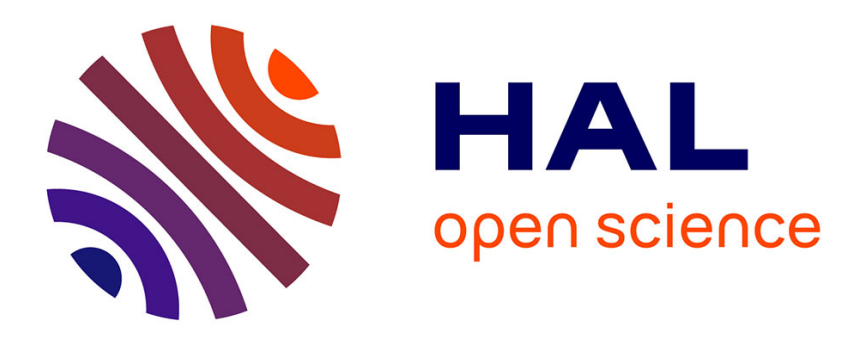

\title{
Body mass-corrected molecular rate for bird mitochondrial DNA
}

Benoit Nabholz, Robert Lanfear, Jérome Fuchs

\section{To cite this version:}

Benoit Nabholz, Robert Lanfear, Jérome Fuchs. Body mass-corrected molecular rate for bird mitochondrial DNA. Molecular Ecology, 2016, 25 (18), pp.4438 - 4449. 10.1111/mec.13780 . hal-01387904

\author{
HAL Id: hal-01387904 \\ https://hal.science/hal-01387904
}

Submitted on 26 Oct 2016

HAL is a multi-disciplinary open access archive for the deposit and dissemination of scientific research documents, whether they are published or not. The documents may come from teaching and research institutions in France or abroad, or from public or private research centers.
L'archive ouverte pluridisciplinaire HAL, est destinée au dépôt et à la diffusion de documents scientifiques de niveau recherche, publiés ou non, émanant des établissements d'enseignement et de recherche français ou étrangers, des laboratoires publics ou privés. 


\section{Body mass-corrected molecular rate for bird mitochondrial DNA}

*Institut des Sciences de l'Evolution, UMR 5554, Université Montpellier place Eugène Bataillon, 634095 Montpellier Cedex 5, France

$\uparrow$ Department of Biological Sciences, Faculty of Science, Macquarie University, NSW 2109, Australia

8 Institut de Systématique, Évolution, Biodiversité, ISYEB-UMR 7205 CNRS, MNHN, UPMC, EPHE; Muséum National d'Histoire Naturelle, Sorbonne Université, 57 rue Cuvier, CP5175005, Paris, France

10

12

Keywords : Mitochondrial, Molecular clock, Birds, body mass

14 Correspondance : Benoit Nabholz, E-mail : benoit.nabholz@umontpellier.fr

Running title : body mass corrected clocks for birds

16

18 


\section{Abstract}

Mitochondrial DNA remains one of the most widely used molecular markers to reconstruct the

26 phylogeny and phylogeography of closely-related birds. It has been proposed that bird mitochondrial genomes evolve at a constant rate of $\sim 0.01$ substitution per site per Million years, i.e. that they evolve

28 according to a strict molecular clock. This molecular clock is often used in studies of bird mitochondrial phylogeny and molecular dating. However, rates of mitochondrial genome evolution

30 vary among bird species, and correlate with life-history traits such as body mass and generation time. These correlations could cause systematic biases in molecular dating studies that assume a strict

32 molecular clock. In this study, we overcome this issue by estimating corrected molecular rates for birds. Using complete or nearly complete mitochondrial genomes of 475 species, we show that there are

34 strong relationships between body mass and substitution rates across birds. We use this information to build models that use bird species' body mass to estimate their substitution rates across a wide range of 36 common mitochondrial markers. We demonstrate the use of these corrected molecular rates on two recently-published datasets. In one case, we obtained molecular dates that are twice as old as the

38 estimates obtained using the strict molecular clock. We hope that this method to estimate molecular rates will increase the accuracy of future molecular dating studies in birds.

40

\section{Introduction}

42 Mitochondrial DNA is widely used to reconstruct the timescale of bird evolution using molecular dating. The divergence times obtained from this approach have been used to examine the impact of life

44 history, geology, climate, and biogeography on the diversification of birds (e.g., Avise \& Walker 1998; Weir et al. 2009; Lanfear et al. 2010). However, the molecular dating methods used to derive the

46 timescale of bird evolution remain controversial.

Most molecular dating studies use a combination of fossil calibrations and relaxed molecular clocks to

48 infer divergence times from molecular data. Fossil calibrations provide information on the divergence times of some nodes in the phylogeny, and relaxed molecular clocks allow divergence times of other

50 nodes to be estimated while allowing for variation in substitution rates among lineages. However, the

Page 2 / 26 
fossil record is scarce or completely lacking for most bird families and genera (Mayr 2009, 2013).

52 Consequently, molecular dating studies in birds often rely on strict molecular clocks, assuming a single substitution rate that does not (or to a limited extent) vary over time and among lineages. This approach

54 allows molecular dates to be estimated entirely in the absence of fossil calibrations. The most frequently employed method is the use of a divergence rate of $2 \%$ per million years (Myr). This rate

56 was proposed some time ago (Brown et al. 1979; Shields \& Wilson 1987; Fleischer et al. 1998), and recently reaffirmed by Weir \& Schluter (2008) in a widely cited analysis in which they estimated a

58 divergence rate of $2.1 \%$ per million years (0.0105 subst/site/myr) for mitochondrial Cytochrome $b$ sequences in birds.

60 Although convenient, the use of strict molecular clocks in birds remains controversial (Lovette 2004; García-Moreno 2004; Pereira \& Baker 2006; Ho 2007; Nabholz et al. 2009; Nguyen \& Ho 2016).

62 Widespread variation in rates of molecular evolution among species is not only predicted on empirical grounds (e.g. Lehtonen \& Lanfear 2014), but has also been repeatedly confirmed in empirical studies of

64 birds (Pereira \& Baker 2006; Nabholz et al. 2009; Eo \& DeWoody 2010; Pacheco et al. 2011; Jarvis et al. 2014; Nguyen \& Ho 2016). Indeed, the broader picture is that variation in substitution rates is the 66 rule rather than the exception across the tree of life (Bromham et al. 1996; Welch et al. 2008; Nabholz et al. 2008; Thomas et al. 2010; Lourenço et al. 2013; Qiu et al. 2014). Failing to account for this

68 variation may lead to systematically biased estimates of divergence times in molecular dating studies. For example, if large birds tend to evolve more slowly than smaller birds, then the use of the 'standard' 70 strict molecular clock would lead to underestimates of divergence times for largest birds and over-estimates of divergence times for smallest birds.

72 Across clades as diverse as vertebrates and angiosperms, substitution rates have repeatedly been shown to correlate with life-history traits such as body mass, longevity, and generation time (Bromham 2009).

74 In birds, it has been shown that substitution rates in both the nuclear and mitochondrial genomes correlate with life history traits (Nabholz et al. 2009; Jarvis et al. 2014) and species richness (Lanfear

76 et al. 2010; Eo \& DeWoody 2010). These correlations suggest that the variation in substitution rates among bird species is, to some extent at least, predictable. This presents opportunities to circumvent the 78 problem of using strict molecular clocks, because one might be able to use commonly measured life-history traits, such as body size, to estimate variation in substitution rates among species. Using

80 this approach, one could define a 'corrected' molecular clock that accounts for some of the known variation in substitution rates among lineages, even in the absence of fossil calibrations.

Page 3 / 26 
In this study, we used the complete or nearly mitochondrial genome of 475 birds species to produce a

84 collection of corrected molecular rates for birds. We focus on body mass because it is known for most extant species of bird (Dunning 2007), and so provides the most convenient life-history trait from

86 which to estimate corrected molecular rates for molecular dating studies. Conveniently, body mass could also be extrapolated using allometric relationship with skeletal measurements (e.g. Campbell and

88 Marcus 1992; Field et al. 2013). We provide corrected molecular rates rates for third codon-positions, as well as for total substitution rates for each of the individual mitochondrial genes that are commonly

90 used for molecular dating in birds. We hope that these corrected molecular rates will increase the accuracy of future molecular dating studies in birds.

92

\section{Materials \& Methods}

94

\section{Sequences, Alignments and Topology}

96 We downloaded all of the complete or near-complete mitochondrial genomes available on NCBI/GenBank (http://bcbi.nlm.nih.gov) in May 2015, resulting in a dataset for 487 species. When

98 multiple individuals were available per species, we selected the longest and/or the highest quality sequence (as inferred from the number of uncertainty codes). When all sequences were deemed to be of

100 equal quality, we selected an individual randomly. We aligned the $12 \mathrm{H}$-stranded protein-coding genes (i.e. all the protein-coding genes except NADH-dehydrogenase 6, ND6) using BioEdit (Hall 1999),

102 after excluding all nucleotides from the stop codons. We carefully checked all alignments by eye. We excluded 12 dubious sequences during alignment, resulting in alignments of 475 species (see Table S1).

104 We estimated a Maximum Likelihood (ML) topology using RAxML (Model GTR+G; Stamatakis 2014). We used a constrained topology to follow Prum et al. (2015) (using option "-g" in RAxML)

106 regarding the basal Neoavians relationships. We also repeated our analyses using a topology following Jarvis et al. (2014) that gave similar results. The topology and the alignments are available at DRYAD 108 DOI. 


\section{Life-history traits}

We obtained body mass data from Dunning (2007). When body mass was not available for a species,

112 we used the median body mass of the genus in Dunning (2007) except if another species of the same genus was available in our dataset. Otherwise, we left that species' body mass unknown. For example,

114 body mass for the black-browed bushtit (Aegithalos bonvaloti) was not present in Dunning (2007) but we left its body mass as unknown in our dataset because another Aegithalos (the long-tailed bushtit,

116 Aegithalos caudatus), for which a mass is available, is present in our dataset. Using this approach, we obtained body mass estimates for 435 of the 475 species in our alignments.

\section{Substitution rate estimation}

120 In order to derive the most accurate molecular rate estimates across species, we used a two-step approach inspired by Nabholz et al. (2008, 2009). This approach allows us to make the best use of the

122 relatively few and relatively old reliable fossil calibrations in birds (Benton et al. 2009; Ksepka \& Clarke 2015), while still estimating rates of evolution for fast-evolving mitochondrial DNA sequences

124 which typically would not be used together with such old fossil calibrations. First, we estimated molecular divergence dates using amino-acid alignment and fossil-based calibrations (see "Molecular

126 dating"). Second, we used molecular divergence dates to calibrate the species-specific substitution rate of nucleotide sequences. An outline of the method are presented in Supplementary Figure S1.

128 To limit the effect of saturation, we defined monophyletic groups of sequences for which the maximal nucleotide divergence between any given pair of species was lower than 0.4 subst./site. To do this, we

130 used a custom R script built with the APE package (Popescu et al. 2012; R Core Team 2013), which led to 84 independent monophyletic clades containing a total of 436 species. Inspection of a saturation plot

132 (a plot of phylogenetic divergences against the uncorrected pairwise genetic divergences) indicated that saturation was moderate at 0.4 subst./site. A threshold set at 0.3 subst./site gave similar results.

134 Within each group, substitution rates were estimated using MCMCTREE (Yang \& Rannala 2006; Rannala \& Yang 2007) implemented in PAML4 (Yang 2007). We applied an HKY model of

136 substitution with an autocorrelated relaxed clock model. We choose the HKY model because it is the most complex model currently implemented in MCMCTREE. The analysis of each group was

138 calibrated using three estimates of the divergence date of the most recent common ancestor of each 
group obtained from the molecular dating analysis based on amino-acid sequences (see below): the

140 mean divergence date; the minimum 95\% credibility interval of the divergence date; and the maximum 95\% credibility interval of the divergence date. Using these three dates allows us assess the effects of

142 the uncertainty of our divergence dates on our molecular rate estimates. The maximum 95\% C.I.'s provide minimal age estimates and therefore maximal substitution rate estimates for each group, while

144 the minimum 95\% C.I.'s provide maximal age estimates and therefore minimal substitution rate estimates for each group. Because several groups contained only two sequences, we added an outgroup

146 to all clades defined as the most closely related species in the phylogeny. For example, a clade contained the two Halcyon species (Halcyon pileata, Halcyon sancta) and we used the Pied Kingfisher

148 (Ceryle rudis) sequence as outgroup. This outgroup was used to help in the estimation of substitution rates for ingroup sequences. Substitution rate estimates for outgroups were not used in downstream

150 analyses, because they were occasionally quite divergent from ingroup sequences and so prone to issues associated with saturation.

\section{Molecular dating}

154 The molecular dating analyses using amino-acid sequences were performed with PHYLOBAYES version 4.1 (Lartillot et al. 2009) using the default site-heterogeneous mixture model (CAT) and a

156 log-normal autocorrelated relaxed clock (option -ln) (Thorne et al. 1998). Autocorrelated relaxed clocks have been shown to outperform other relaxed-clock models (Lepage et al. 2007). The priors on

158 divergence dates were uniform (Lepage et al. 2007). We ran two chains in parallel for at least 20000 steps for all the analyses, with a burn-in of 10000 steps. In all cases, the two chains gave similar results,

160 suggesting that the MCMC analyses had converged. Convergences was also assessed visually and using "tracecomp" ensuring that the discrepancy statistic $d$ between estimates parameters were below

1620.4 for most parameters.

For the calibrations, we relied exclusively on fossils, in order to avoid potential problems linked to 164 calibrations based on geographic distributions (Heads 2005, 2011). We particularly avoided the controversial calibration based on the vicariance of New-Zealand to calibrate the first split within the

166 passeriformes (Trewick \& Gibb 2010; Mayr 2013; Gibb et al. 2015). Although less controversial, more recent biogeographic calibrations have been used to date the diversification of the Hawaiian 168 honeycreepers (Lerner et al. 2011). We chose to not use these calibrations but instead use them as an 
independent source of information to evaluate our analyses.

170 As the placement of some calibrations is ambiguous, we choose to test several combinations of fossil calibrations summarized in Table 1, and discuss specific cases here. The Anseriformes fossil Vegavis

172 iaai, found in the Maastrichtian, would seem to provide a clear minimum bound for the Neognathae/Paleognathae split (Benton et al. 2009; Ksepka \& Clarke 2015). However, we chose to not

174 consider Vegavis iaai as an Anseriformes because its phylogenetic placement is ambiguous (Mayr 2009, 2013). For the maximum bound of the Neognathae/Paleognathae split, we followed the

176 proposition of Benton et al. (2009) and set it at 86.5 Myr. Alternatively, Gansus yumenensis from the early Cretaceous of China (110 Myr) was used by Jetz et al. (2012) whereas Jarvis et al. (2014) used

178 the maximum boundary of the Upper Cretaceous (99.6 Myr).

The other minimum-bound calibrations we used in our molecular dating analysis all followed the 180 suggestions of Ksepka \& Clarke (2015). Additionally, we constrained the Passeriformes/Psittaciformes split between 53.5 and 65.5 Myr based on the first Eocene fossil of Australia (Boles 1997) and on the 182 stem Psittaciformes fossil Pulchrapollia gracilis (Prum et al. 2015). The maximum bound at 65.5 Myr was used in all analyses except calibration set 1 (see Table 1) (Longrich et al. 2011; Prum et al. 2015) .

184 Finally, in one analysis (calibration set 4), we constrained the Oscines/Suboscines split between 28 and 34 Myr based on the first Suboscines from early Oligocene (28Myr, Mayr \& Manegold 2006) and on 186 the Eocene/Oligocene limit (34Myr). This later maximum bound relies on the fact that all Passeriformes fossils known from the Eocene are stem Passeriformes, therefore crown Oscines and 188 Suboscines may have appeared latter during the Oligocene (Mayr \& Manegold 2004; Mayr 2013).

Table 1: Fossil calibration combinations used in molecular dating analyses

\begin{tabular}{ccccc}
\hline Calibration Sets & Taxon 1 & Taxon 2 & $\begin{array}{c}\text { Maximum bound } \\
(\mathrm{Myr})\end{array}$ & $\begin{array}{c}\text { Minimum bound } \\
(\mathrm{Myr})\end{array}$ \\
\hline $1,2,4$ & Neognathae & Paleognathae & 86.5 & 66 \\
3 & Neognathae & Paleognathae & 110 & 66 \\
$1,2,3,4$ & Anseriformes & Galliformes & free & 66 \\
$1,2,3,4$ & Sphenisciformes & Procellariiformes & free & 60.5 \\
$1,2,3,4$ & Coraciidae & Alcedinidae & free & 51.57 \\
$1,2,3,4$ & Apodidae & Trochilidae & free & 51 \\
1 & Psittaciformes & Passeriformes & free & 53.5
\end{tabular}



$2,3,4$
Psittaciformes
Passeriformes
65.5
53.5
4
Oscines
Suboscines
34
28

In order to compare the results obtained using the four different calibration sets we propose (Table 1),

192 we selected five divergence dates within the phylogeny (Table 2) for which we have independent evidence with which to assess the accuracy of our own analyses: (i) the first divergence within the

194 extant Hawaiian honeycreeper which was inferred to occur 6 Myr (Lerner et al. 2011); (ii) the Sittidae (represented by Sitta) / Troglodytidae (represented by Henicorhina and Campylorhynchus) split for

196 which the maximum bound is provided by the early Miocene (20.5-18 Myr) fossil of a Certhioidea (a clade containing the Sittidae and Certhidae) (Manegold 2007); (iii) the Oscines/Suboscines split for

198 which a maximum bound is provided by the first Suboscines fossils ( 28 Myr) (Mayr \& Manegold 2006); (iv) the Stercorariidae / Alcidae split; and (v) the Jacanidae / other Scolopaci split; for both (iv)

200 and (v) Smith (2015) provides minimum calibrations points that could be compared with our analyses (Table 2).

202

\section{Corrected rate model}

204 All the statistical analyses were performed with R (R Core Team 2013). In the linear regression, body mass and substitution rate were log transformed (logarithm of base 10) and parameters of the linear

206 model were estimated using the $\mathrm{R}$ formula $\operatorname{lm}(\log 10$ (Rate) $\sim \log 10$ (Mass)). We repeated this analysis accounting for the relatedness among species using phylogenetic generalised least squares (PGLS)

208 (Grafen 1989; Garland et al. 2000), and phylogenetic independent contrast (PIC) (Felsenstein 1974), implemented in APE (Paradis 2011). However, the parameters estimated using these methods could

210 not be used to obtain corrected substitution rates using an independent species' body mass.

\section{Substitution rate variation among protein-coding genes}

In order to test for variation in substitution rates among different protein-coding genes, we performed

214 additional molecular dating analyses for all genes individually, except for the very short ATP8 and ND4L genes. This led to an additional 20 molecular dating analyses: all codon positions and third 216 codon positions for each of 10 protein-coding genes individually. For each of these analyses, we 
estimated the branch lengths using the F84 model implemented in BASEML (Yang 2007). We did not

218 use the HKY model here because we could not optimize it using BASEML on some datasets. However, the F84 model is conceptually similar to the HKY model used in MCMCTREE analyses. To avoid 220 overparametrisation due to the short alignments, we fixed the value of the Kappa parameter (the transition to transversion ratio) to the value estimated using the whole dataset (Kappa $=8$ ). We also

222 used the gamma distribution of rate variation among sites only in the analyses using all codon positions. We obtained substitution rates by dividing the terminal branch lengths by the appropriate

224 divergence times estimated in the molecular dating analyses. In this case, very small branches can be associated with extremely high variance, we therefore excluded branch lengths shorter than 0.01

226 substitution per site. The same sets of species were analyzed for every gene meaning that if a species has a branch shorter than 0.01 for on particular gene it was excluded for all, leading a set of $\mathrm{N}=292$

228 species. We did not use MCMCTREE for these analyses because many of these individual-gene datasets were small and the MCMC chain failed to converge.

230 To statistically compare substitution rates among protein-coding genes, we used an ANOVA with single factor repeated measures design with a model reflecting that the genes are nested within the 84 232 independent monophyletic clades (R formula : $\operatorname{lm}($ Rate $\sim$ Gene + Gene:Clade) ). We also tested a model were Clade was a random effect ( $\mathrm{R}$ formula : $\operatorname{lme}($ Rate $\sim$ Gene, random $=\sim 1 \mid$ Clade/Gene)).

234 Finally, we tested the rate differences between each pair of genes using pairwise t-tests with adjusted p-values using a Bonferroni correction.

\section{Working examples}

238 We applied our method to two recently published datasets including relatively large and small birds. The first datasets was composed of 16 ND1 sequences of Circus Harriers (body mass 500g; Oatley et

240 al. 2015). For this analysis, we used the phylogenetic tree estimated from these sequences in the original study (Figure 2 of Oatley et al. 2015).

242 The second dataset was composed of CYTB and ND2 sequences of the Buff-throated Woodcreeper complex (genus Xiphorhynchus, body mass $\sim 40 \mathrm{~g}$; Rocha et al. 2015). We restricted the analysis to one

244 sequence per taxon (as presented in Appendix A and Appendix B of Rocha et al. 2015) leading to a total of 11 sequences. As above, we used the phylogenetic tree estimated from these sequences in the 
original study (Figure 1 of Rocha et al. 2015).

In both examples, sequences were aligned using muscle (Edgar 2004) and checked by eye. Sites 248 containing missing data were excluded. Branch lengths were estimated exclusively from the third codon position. We applied a molecular clock model in BASEML (model F84, Kappa parameter fixed

250 to 8.0 , parameter "clock $=1$ "). Then we scaled the branch lengths of the tree using the body mass corrected substitution rate for each clade (i.e. a single scalar is used for the entire tree). Finally, we

252 estimated branch lengths from all codon positions for both datasets in order to compare our estimates with the commonly-used 2\% per Myrs strict molecular clock rate. Alignments, phylogenies and the R 254 script to convert branch lengths to divergence dates are provided at DRYAD.

\section{Results}

\section{Molecular dating}

258 Most fossil-based calibrations available are relatively deep in the bird phylogeny (Benton et al. 2009; Jarvis et al. 2014; Ksepka \& Clarke 2015; Prum et al. 2015), and are not suited to the fast evolving 260 nucleotide sequences of the mitochondrial genome (Nguyen \& Ho 2016). To make use of these ancient calibrations, we performed a molecular dating analysis with the amino-acid sequences because they are

262 less prone to saturation (Brown et al. 1982). We used four sets of calibrations (Table 1) reflecting the current uncertainty surrounding fossil calibrations for molecular dating analyses of birds. We also

264 tested two topologies reflecting alternative hypotheses regarding the basal Neoavian divergences (Jarvis et al. 2014; Prum et al. 2015). We obtain very similar dates between topologies (results not

266 shown). Hereafter, we present only the results obtained using the Prum et al. (2015) topology.

Globally, the calibration sets 1, 2 and 3 provide very similar divergence dates that are all compatible

268 with independent fossil data (Table 2). The Oscines/Suboscines divergences are, however, 30 to $35 \mathrm{Myr}$ older that the first known fossils (Mayr \& Manegold 2004; Mayr 2013). Our analyses also provide

270 divergence dates that are more than twice as old as the dates of Lerner et al. (2011) for the diversification of Hawaiian honeycreepers (Table 2).

272 Table 2: Comparison of molecular dating analyses with independent fossils or independent analysis. Mean dates are indicated and $95 \%$ C.I. are provided within brackets.

Page 10 / 26 


\begin{tabular}{cccccc}
\hline $\begin{array}{c}\text { \# Calibration } \\
\text { sets }\end{array}$ & $\begin{array}{c}\text { Hawaiian } \\
\text { honeycreepers }\end{array}$ & Oscines / Suboscines & $\begin{array}{c}\text { Sittidae / } \\
\text { Troglodytidae }\end{array}$ & $\begin{array}{c}\text { Stercorariidae / } \\
\text { Alcidae }\end{array}$ & $\begin{array}{c}\text { Jacanidae / other } \\
\text { Scolopaci }\end{array}$ \\
\hline $\begin{array}{c}\text { Minimum } \\
\text { fossil bound } \\
\text { (Myr) }\end{array}$ & 6 & 28 & 18 & 34.2 & 30 \\
1 & 15.8 & & & & 41.8 \\
& $(12.1,19.9)$ & $(57.8,69.9)$ & $(29.9,40.6)$ & $(39.6,56.5)$ & $(32.2,51.4)$ \\
2 & 13.4 & 57.1 & 30.3 & 43.9 & 37.6 \\
3 & $(10.1,16.9)$ & $(54.9,59.1)$ & $(26.7,33.6)$ & $(35.8,49.4)$ & $(28.7,46.0)$ \\
& 14.3 & 57.4 & 31.6 & 42.7 & 36.9 \\
$4^{* *}$ & $(10.9,18.0)$ & $(55.1,59.3)$ & $(27.3,35.3)$ & $(36.4,48.9)$ & $(28.1,45.5)$ \\
& 7.2 & 33.9 & 19.0 & 41.2 & 35.1 \\
& $(5.8,8.8)$ & $(33.5,34.0)^{* *}$ & $(\mathbf{1 7 . 3 *}, 21.0)$ & $(34.9,46.5)$ & $(\mathbf{2 6 . 6 *}, 42.3)$ \\
\hline
\end{tabular}

$274 *$ Indicates values that are in conflict with the fossil records.

**This analysis, the Oscines / Suboscines split was constrained to occur between 28 and $34 \mathrm{Myr}$

276

Calibration set 4 provides younger dates than the other calibration sets, particularly within the

278 Passeriformes. This is expected as this analysis includes an additional calibration bound that constrained Oscines / Suboscines split to occur between 28 and 34 Myr. This is about half the age of

280 this split recovered with other calibration sets that do not calibrate this node (Table 2). This analysis provided dates that are in agreement with those of Lerner et al. (2011) but the minimum 95\%

282 credibility intervals (C.I.) are younger than the fossils records for the Sittidae / Troglodytidae split and for the Jacanidae / other Scolopaci split although the discrepancy is modest (Table 2, 17.3 vs $18 \mathrm{Myr}$ 284 and 26.6 vs 30 Myr respectively).

It is difficult to favor one analysis over the others, therefore, we choose to consider both calibration set 2862 (which is representative of the set 1,2 and 3) and calibration set 4 for the following analyses.

\section{A mass-corrected molecular rate}

We selected 84 independent monophyletic groups containing a total 437 species (Table S2). These 290 groups match a criterion of limited divergence in order to accurately estimate substitution rates. Substitution rates were calibrated for each group using divergence dates obtained from the molecular 292 dating using the fossil calibration sets 2 and 4 (see method). Hereafter, we will refer to them as 
“analysis 2" and "analysis 4" respectively.

294 For analysis 2 , the median third codon substitution rate was $0.0142 \mathrm{subst} / \mathrm{sites} / \mathrm{Myr}(\mathrm{min}=0.0035$, $\max =0.0306$ ). For analysis 4 , the median third-codon substitution rate was $0.0165 \mathrm{subst} / \mathrm{site} / \mathrm{Myr}$ (min $296=0.0042, \max =0.0566)$. In both cases, the substitution rate varies by more than on order of magnitude among species.

298 To compare our approach with the widely-used divergence rate of 0.01 substitutions per site per million years, which was originally estimated from all codon positions, we also inferred substitution rates using

300 all codon positions. For analysis 2, the median substitution rate was $0.0035 \mathrm{subst} / \mathrm{site} / \mathrm{Myr}(\mathrm{min}=$ 0.0010, $\max =0.0090$ ) with all species having a rate below 0.01. For analysis 4, the median

302 substitution rate was $0.0042 \mathrm{subst} / \mathrm{site} / \mathrm{Myr}$ but the distribution obtained overlapped extensively with the $0.01(\min =0.0011, \max =0.0158)$. The passerines, for example, have a median substitution rate 304 that is very close to it (median $=0.009 \mathrm{subst} / \mathrm{site} / \mathrm{Myr}$ ).

Both third codon and all position substitution rates are strongly negatively correlated with body mass

306 (Table 3 ). The $\mathrm{R}^{2}$ values are higher when substitution rates are estimated from third-codon positions ( 0.46 and 0.60 for the analysis 2 and 4 respectively, Figure 1$)$ than when they are estimated from all 308 codon positions ( 0.35 and 0.51 for the analysis 2 and 4 respectively). We speculate that this could result from the effects of natural selection on substitution rates. These effects are likely to be stronger on

310 codon positions 1 and 2 than on third codon positions, and may weaken the relationship between body mass an substitution rates. However, regardless of the cause of the higher $\mathrm{R}^{2}$ for third codon positions,

312 it implies that body mass is a better predictor of third codon position substitution rates than of substitution rates derived from all codon positions. As such, body mass corrected rates will be more

314 accurate if they are derived using substitution rates estimated from third codon positions only, and so we focus on deriving corrected rates for third codon positions only.

316 Both the GLS and PIC analyses, which control for phylogenetic non-independence among species, confirmed a significant negative correlation between substitution rate and body mass (Table S3),

318 demonstrating that the relationship between body mass and substitution rate is robust to control of the phylogenetic relationship among species. A similar conclusion was made by Nabholz et al. (2009).

320 The slope and the intercept of the linear models comparing third codon position substitution rates and body mass are presented in Table 3. These models allow us to predict a species' third codon 322 mitochondrial substitution rate given its body mass using the following equation:

Page 12 / 26 


$$
\text { Substitution rate }=\frac{10^{(\text {slope } * \log 10(\text { Mass })+\text { intercept })}}{100}
$$

324 For example, using the parameters of analysis 2, a bird of $10 \mathrm{~g}$ (e.g. small passerines - some White Eyes: Zosteropidae, Sunbirds: Nectariniidae) is predicted to evolve at $\left(10^{(-0.145 * \log 10(10)+0.459)} / 100\right) 0.0206$

326 subst/site/Myr for third codon position. In contrast, a birds of $1000 \mathrm{~g}$ such as the Mallard (Anas platyrhynchos), the Kelp gull (Larus dominicanus) or a medium size raptors such as the Crested

328 Serpent Eagle (Spilornis cheela) for example, will evolve 2 times slower at 0.0106 subst/site/Myr for third codon position. These values convert to divergence rates of $4.2 \%$ and $2.1 \%$ per Myr respectively.

330 Analysis 4 gave linear models with steeper slopes than analysis 2, which creates a larger difference between the predicted substitution rates for any given pair of species. For example, in Analysis 4, birds

332 of $10 \mathrm{~g}$ and $1 \mathrm{~kg}$ are predicted to have substitution rates of 0.0368 and 0.0118 subst/site/Myr respectively ( $7.4 \%$ and $2.4 \%$ divergence per Myr).

334 Finally, to take the uncertainty of molecular dating into account, we repeated the substitution rate estimation using secondary calibration points taken from the minimum and the maximum $95 \%$ C.I. of 336 the estimated divergences dates. The maximum 95\% C.I. provide minimal divergence and, therefore, an estimated maximum for the substitution rate whereas the minimum $95 \%$ C.I. dates provide a minimal 338 substitution rate (Table 3$)$.

340 Table 3: Parameter of the linear model: $\log 10$ (Rate) $\log 10$ (body mass). Substitution rates were divided by 100 in the model. Maximum and minimum 95\% C.I. correspond of estimates made using 95\% C.I.

342 of molecular dating.

\begin{tabular}{cccccc}
\hline Positions & $\begin{array}{c}\text { Calibration } \\
\text { sets }\end{array}$ & $\mathrm{R}^{2}$ & $\begin{array}{c}\text { Mean } \\
\text { slope; intercept }\end{array}$ & $\begin{array}{c}\text { Maximum 95\% CI } \\
\text { slope; intercept }\end{array}$ & $\begin{array}{c}\text { Minimum 95\% CI } \\
\text { slope; intercept }\end{array}$ \\
\hline $3^{\text {rd }}$ & 2 & 0.46 & $-0.145 ; 0.459$ & $-0.143 ; 0.559$ & $-0.141 ; 0.367$ \\
$3^{\text {rd }}$ & 4 & 0.60 & $-0.247 ; 0.813$ & $-0.243 ; 0.905$ & $-0.246 ; 0.730$ \\
All & 2 & 0.35 & $-0.122 ;-0.185$ & $-0.123 ; 0.076$ & $-0.118 ;-0.274$ \\
All & 4 & 0.51 & $-0.227 ; 0.177$ & $-0.220 ; 0.266$ & $-0.224 ; 0.089$ \\
\hline
\end{tabular}

\section{Substitution rates differ among Protein-coding genes}

For each of the protein-coding genes in our dataset (except ND6, ND4L and ATP8), we estimated 
346 substitution rates from both third codon positions alone and all codon positions combined. The results from analyses 2 and 4 are qualitatively similar, so we only present the results from analysis 2 here.

348 Using all codon positions, we detected significant variation in substitution rates among genes (linear model, Gene effect : p<0.001). COX1, COX2, COX3 and CYTB have a lower substitution rate than the

350 ND genes and ATP6 (Figure 2a, pairwise t-test). The fastest-evolving gene (ND2, median rate = $0.0081)$ evolves, on average, 1.5 faster than the slowest (COX2, median rate $=0.0055)$ (see Table S3

352 for details).

Using third codon positions, we also detected significant variation in substitution rates among genes

354 (linear model, Gene effect : $\mathrm{p}<0.001$ ). As for all codon positions, COX proteins are generally slower than ND and ATP6 (Figure 2b). The range of variation is lower, with the fastest gene (ND1, median

356 rate $=0.0150)$ evolving 1.2 faster than the slowest $(\mathrm{COX} 3$, median rate $=0.0121)$.

To take these variations into account, we computed the difference between the median rate of each gene 358 individually with the full alignment (Table S4). These values could serve as correction factor to the mass-corrected substitutions when using a reduced sets of genes.

\section{Working examples}

\section{Circus Harriers}

We obtained the body mass of 14 out of 16 species (C. hudsonius and C. spilothorax are missing) of

364 harriers in the original dataset. body mass appears homogeneous in this clade $($ mean $=507 \mathrm{~g}, \mathrm{sd}=132 \mathrm{~g})$ which is ideal to apply a single mass-corrected rate for the whole phylogeny. Moreover, the molecular

366 divergence is moderate, with the maximal divergence between any two species at 14\%. It is important to limit the divergence as third codon position can saturate rapidly due to their fast rate of evolution,

368 leading to an inaccurate branch length estimates (Nguyen \& Ho 2016). Using the parameters from Table 3, the mass-corrected substitution rates vary between $0.0116(0.0097-0.0149)$ subst/sites/Myr

370 for third codon position and $0.0140(0.0116-0.0177)$ subst/sites/Myr for third codon position for the calibration sets 2 and 4 respectively. This rate leads to divergence dates that are approximately twice as

372 old as the dates that would be obtained by assuming the widely-used molecular clock rate of $2.1 \%$ divergence per Myr (Table 4). The first split within the genus (Node B) is estimated to have occurred at 37410.3 to 15.8 Myr following by the diversification of "steppe" Harrier (Node C, D, and I, Oatley et al. 
2015). Applying the gene specific rate, the discrepancy would have been even greater because ND1

376 evolves 5-7.5\% faster than the rate estimated with all the protein-coding genes (Table S3). Although much older, these dates are still largely in agreement with the appearance of open habitat and C4 378 grasslands ecosystems during mid-Miocene (Edwards et al. 2010). Our estimates also support a recent origin of the Marsh harrier clades (Node E) of between 2.9 and 4.4 Myr.

380 Table 4: Divergence times and confidence intervals according to body mass corrected substitution rate (Calibration sets 2 and 4) and to the $2.1 \%$ of divergence per Myr. The node are presented in the figure 2 382 of Oatley et al. (2015)

\begin{tabular}{cccc}
\hline Node & $2.1 \%( \pm 0.1 \%)$ & Calibration set 2 & Calibration set 4 \\
\hline B & $6.3(6-6.6)$ & $13.1(10.3-15.8)$ & $11(8.7-13.2)$ \\
C & $4.7(4.5-4.9)$ & $9.7(7.6-11.6)$ & $8.1(6.4-9.7)$ \\
D & $4.2(4-4.4)$ & $8.3(6.5-10.1)$ & $7(5.5-8.4)$ \\
E & $2(1.9-2.1)$ & $3.7(2.9-4.4)$ & $3.1(2.4-3.7)$ \\
F & $1.0(0.9-1)$ & $1.7(1.3-2.0)$ & $1.4(1.1-1.7)$ \\
G & $0.5(0.5-0.6)$ & $0.8(0.6-0.9)$ & $0.6(0.5-0.8)$ \\
H & $0.1(0.1-0.1)$ & $0.1(0.1-0.2)$ & $0.1(0.1-0.1)$ \\
I & $5.5(5.2-5.7)$ & $12(9.4-14.5)$ & $10.1(7.9-12.1)$ \\
J & $1.7(1.7-1.8)$ & $3.5(2.7-4.2)$ & $2.9(2.3-3.5)$ \\
K & $1.3(1.2-1.4)$ & $2.5(1.9-3)$ & $2.1(1.6-2.5)$ \\
L & $0.9(0.9-1)$ & $2.0(1.5-2.4)$ & $1.6(1.3-2)$ \\
\hline
\end{tabular}

\section{Buff-throated Woodcreeper complex Xiphorhynchus}

We obtained the body mass of 6 out of 7 species of Xiphorhynchus (X. eytoni is not available) in the 386 original dataset. The maximal molecular divergence between any two species is $12 \%$. As for the Harrier, the body mass appears very homogeneous in this clade (mean $=48.6 \mathrm{~g}, \mathrm{sd}=8.8 \mathrm{~g}$ ). Using the 388 parameters from Table 3 , the mass-corrected substitution rates vary between $0.0163(0.0135-0.0208)$ subst/sites/Myr for third codon position and 0.0249 (0.0207-0.0313) subst/sites/Myr for third codon 
390 position using for calibration sets 2 and 4 respectively. The mass-corrected rate leads to divergence dates that are close to those obtained using the widely-used divergence rate of $2.1 \%$ per Myr (Table 5)

392 and, therefore, are largely congruent with the dates proposed in the original publication that include paleontological and fossil calibrations as well as the molecular clock calibration (Rocha et al. 2015).

394 Table 5: Divergence times and confidence intervals according to body mass corrected substitution rate (Calibration sets 2 and 4) and to the $2.1 \%$ of divergence per Myr. The node are presented in the figure 3 396 of Rocha et al. (2015)

\begin{tabular}{cccc}
\hline Node & $2.1 \%( \pm 0.1 \%)$ & Calibration set 2 & Calibration set 4 \\
\hline I & $3.9(3.8-4.1)$ & $6.0(4.7-7.3)$ & $3.9(3.1-4.7)$ \\
II & $3.4(3.2-3.6)$ & $5.4(4.2-6.5)$ & $3.5(2.8-4.2)$ \\
III & $2.2(2.1-2.3)$ & $3.1(2.5-3.8)$ & $2.1(1.7-2.5)$ \\
IV & $1.3(1.2-1.3)$ & $2.1(1.7-2.6)$ & $1.4(1.1-1.7)$ \\
V & $0.4(0.4-0.5)$ & $0.6(0.5-0.7)$ & $0.4(0.3-0.5)$
\end{tabular}

\section{Discussion}

In the present study, we propose a simple model to predict mitochondrial substitution rates from 400 species' body mass. In line with several previous studies (Pereira \& Baker 2006; Brown et al. 2008; Nabholz et al. 2009; Lanfear et al. 2010; Eo \& DeWoody 2010; Pacheco et al. 2011), we detected 402 extensive and statistically significant variation in substitution rates among bird species. Using body mass alone, we were able to predict up to $60 \%$ of the variation in observed substitution rates, 404 suggesting that using body mass corrected molecular rates is superior to a strict molecular clock approach.

406 The extensive substitution rate variation we describe is smaller than the one reported by Nabholz et al. (2009). Nabholz et al. (2009) reported a median of 0.018 subst/sites/Myr with a variation from 0.003 408 and 0.09 subst/sites/Myr. This discrepancy could simply be explained by the fact that Nabholz et al. (2009) used only CYTB for molecular dating and substitution rate estimation in contrast to all the

410 H-strand encoded protein-coding genes therefore leading to higher sampling variance in the substitution process in the earlier study.

Page 16 / 26 
412 Our estimates of mitochondrial substitution rates are often lower than the commonly-used divergence rate of $2 \%$ per million years, although, they are largely concordant with the estimates of Pereira and

414 Baker (2006) (range from 0.0009 to 0.012 subst/sites/Myr) and Pacheco et al. (2011) (average rate per genes range from 0.0027 to 0.0068 subst/sites/Myr). It was only in the analysis constraining the

416 divergence between the Oscines and Suboscines to be younger than 34 Myrs (Analysis 4) that a substantial number of species (mostly passerines) have a divergence rates close to or above $2 \%$ per 418 million years. The discrepancy between the analyses reflect the current uncertainty surrounding fossils calibrations used to estimate the timescale of avian diversification (Mayr 2013; Cracraft et al. 2015;

420 Mitchell et al. 2015). For example, analyses with calibration sets 1, 2 and 3 provide an age for the Oscines / Suboscines divergence that is 30 to 35 Myr years older than the fossil record. This 422 discrepancy may seem surprising because no stem Passeriformes are known from the Paleocene, let alone the derived Oscines and Suboscine representatives (Mayr 2009). However, the fossil record of 424 passerines could be biased towards missing the earliest fossils, because most known deposits exist in the northern hemisphere whereas both crown and stem Passeriformes likely originated in the southern

426 hemisphere (Boles 1997; Ericson et al. 2002). Our analyses 1, 2, and 3 suggest that the diversification of Hawaiian Honeycreepers predate the oldest Hawaiian island (Kauai-Miihau). Thus, our analyses 428 support either that the diversification started before the emergence of the oldest island or, that at least two independent colonization events have occurred. Both scenarios are possible as older islands existed 430 before Kauai-Miihau (Price \& Clague 2002) and many examples of old taxa on young islands are known (Heads 2011), although the latter could also result from the extinction of the mainland sister 432 taxa.

Our analyses show that the correlations between body mass and substitution rate are stronger when 434 substitution rates are estimated exclusively from third codon positions. This could be because third codon positions are less affected by natural selection than first and second codon positions. Selective 436 constraint acting on mitochondrial genes is known to be associated with speed of movement (Shen et al. 2009) and island/mainland status (Johnson \& Seger 2001; Woolfit \& Bromham 2005), a variable

438 related to the effective population size. Positive selection has also been detected in vertebrate mitochondrial evolution (Grossman et al. 2004; Castoe et al. 2008, 2009) although not, to date, in 440 birds. Positive selection could episodically inflate the substitution rate. Regardless of the reason for the difference in the strength of association between body mass and substitution rate estimates, we suggest 442 using molecular rates estimated from third codon positions when using body mass corrected molecular 
rates, since it is these rates which can be most accurately predicted from body mass data. Our analyses

444 reveal significant variation in substitution rates among mitochondrial protein-coding genes. Using substitution rates estimated from all codon positions, we show that NADH-dehydrogenase (ND, 446 proteins of the complex I) genes evolved faster than Cytochrome Oxiydase (COX, proteins of the complex IV) and CYTB genes. These results are in line with those of Eo \& DeWoody (2010), Smith \&

448 Klicka (2010), Pacheco et al. (2011), Lerner et al. (2011) and Nguyen \& Ho (2016). Eo \& DeWoody (2010) and Pacheco et al. (2011) report a variation among genes that is higher than in our analysis (e.g.

450 ND2 being 2.2 times faster than COX1 in Pacheco et al. 2011). Using datasets composed of passerines, Nguyen \& Ho (2016) also detected fast evolution for ND genes, however, CYTB was reported as 452 clearly the slowest genes which is not the case in our analysis. The observed variation might be a consequence of the variation in evolutionary constraint among genes, since the ND genes are known to

454 be less constrained than the COX genes (Mishmar et al. 2003; Nabholz et al. 2013). Interestingly, we also detected extensive variation of third codon position substitution rates among protein-coding genes.

456 The variation among genes could be explained in two ways: i) a difference in mutation rate among genes and ii) selection acting on synonymous sites. Neither GC content nor the positions of the protein 458 in the genome (that is linked to the time spend single stranded during, Reyes et al. 1998) explain the observed difference in substitution rate among genes. Selection on synonymous sites is a possibility, for

460 example, through a selection on codon usage driven by the large variation of expression level among protein-coding genes (Nabholz et al. 2013). Additionally, indirect evidence of selection on synonymous

462 sites is also provided by mutations linked to Human disease. Among the 271 point mutations located within the protein-coding genes that are known to cause diseases in humans, nine are synonymous 464 mutations (Lott et al. 2013).

Finally, we re-analysed two datasets to illustrate the principle of our method. These analyses show that 466 in some cases, particularly with non-passerine birds, the assumption of the widely-used divergence rate of $2 \%$ per million years can give very different answers from using the body mass corrected molecular 468 rates we derive here. We emphasis three additional points as a guideline to users of our corrected molecular rates. First, the species analysed should have a limited molecular divergence. An arbitrary 470 threshold could be a pairwise molecular divergence below 1.0 subst/site for the third-codon position, which corresponds to a divergence of $0.3-0.4 \mathrm{subst} / \mathrm{site}$ (that is 30 to $40 \%$ divergence) for all codon 472 position. When divergence is higher, we recommend splitting the dataset into smaller clades and analysing each clade independently. Second, when few loci are available, over-parametrisation of the 
474 model of molecular evolution could be an issue. Particularly, we noted that the Kappa parameter in our empirical analyses increased to unrealistic values $(>100)$ in some small datasets, leading to

476 unrealistically inflated branch length estimates. We therefore recommend fixing this parameter to a reasonable value a priori - for example, the one estimated using all codon positions - when only a few

478 loci are available for analysis. Third, the within-clade body mass should be rather homogeneous among the species of a clade (this is likely when analysing clades of limited molecular divergence). This will

480 help to ensure that applying a single correction factor the molecular branch-lengths is warranted. If body mass varies widely within a clade, the substitution rate is unlikely to be accurately approximated 482 using a single molecular clock estimated from the average body mass of that clade. In this case, we also recommend splitting the dataset into monophyletic clades of species with relatively homogeneous body 484 mass, and analysing each clade independently.

\section{Acknowledgments}

We thank Loïs Rancilhac and Florent Sylvestre for their help during preliminary analysis. We also 488 thank the four anonymous reviewers for their comments and corrections. This work was supported by Agence Nationale de la Recherche grants ANR-14-CE02-0002-01 "BirdIslandGenomic” to B. N. This 490 is ISEM publication number ISEM 2016-147.

\section{Data accessibility}

- Alignments, phylogeny, and body mass data for the main dataset, the two working examples and R 494 script to compute the molecular date using species body mass : Dryad doi:10.5061/dryad.1040d

\section{Legends}

Figure 1: Correlation between body mass and third-codon position substitution rates. The dotted green 498 line indicates an approximation of the rate corresponding to a $2 \%$ divergence per million years.

Figure 2: Pairwise comparison of substitution rates between protein-coding genes. Colour indicates the 500 direction and the intensity of the difference. A deep blue color indicates that the gene on the X-axis evolves at a faster rate than the gene on the Y-axis whereas a white color indicates the opposite. A star 
502 indicates when the difference is statistically significant (Pairwise t-test with $\mathrm{p}$ value $<0.01$ after Bonferroni correction).

504 Figure S1: Schematic descriptions of two-steps method. A) The molecular dating is performed on the whole phylogeny using amino-acids sequences and fossil-based calibrations; B) The species-specific

506 substitution rate is estimated in groups of moderate divergence (here group \#40) using third-codon positions. The blue dot represents the calibration obtained from analysis $\mathrm{A}$ and used to estimate 508 divergence date in analysis B.

\section{References}

Avise JC, Walker DE (1998) Pleistocene phylogeographic effects on avian populations and the speciation process. Proceedings of the Royal Society of London B: Biological Sciences, 265, $457-463$.

Benton MJ, Donoghue PCJ, Asher RJ (2009) Calibrating and constraining molecular clocks. The timetree of life, 35-86.

Boles WE (1997) Fossil Songbirds (Passeriformes) from the Early Eocene of Australia. Emu, 97, $43-50$.

Bromham L (2009) Why do species vary in their rate of molecular evolution? Biology Letters, 5, 401-404.

Bromham L, Rambaut A, Harvey PH (1996) Determinants of rate variation in mammalian DNA sequence evolution. Journal of Molecular Evolution, 43, 610-621.

Brown WM, George M, Wilson AC (1979) Rapid evolution of animal mitochondrial DNA. Proceedings of the National Academy of Sciences, 76, 1967-1971.

Brown WM, Prager EM, Wang A, Wilson AC (1982) Mitochondrial DNA sequences of primates: Tempo and mode of evolution. Journal of Molecular Evolution, 18, 1432-1432.

Brown JW, Rest JS, García-Moreno J, Sorenson MD, Mindell DP (2008) Strong mitochondrial DNA support for a Cretaceous origin of modern avian lineages. BMC Biology, 6, 6.

Campbell KE, Marcus L (1992) The relationships of hindlimb bone dimensions to body weight in Page 20 / 26 
birds. Natural History Museum of Los Angeles County Science Series, 36.

Castoe TA, Jiang ZJ, Gu W, Wang ZO, Pollock DD (2008) Adaptive evolution and functional redesign of core metabolic proteins in snakes. PloS One, 3, e2201.

Castoe TA, de Koning APJ, Kim H-M et al. (2009) Evidence for an ancient adaptive episode of convergent molecular evolution. Proceedings of the National Academy of Sciences of the United States of America, 106, 8986-8991.

Cracraft J, Houde P, Ho SYW et al. (2015) Response to Comment on "Whole-genome analyses resolve early branches in the tree of life of modern birds." Science, 349, 1460-1460.

Dunning JBJ (2007) CRC Handbook of Avian Body Masses, Second Edition. CRC Press.

Edgar RC (2004) MUSCLE: a multiple sequence alignment method with reduced time and space complexity. BMC Bioinformatics, 5, 113.

Edwards EJ, Osborne CP, Strömberg CAE, Smith SA, Consortium CG (2010) The Origins of C4 Grasslands: Integrating Evolutionary and Ecosystem Science. Science, 328, 587-591.

Eo SH, DeWoody JA (2010) Evolutionary rates of mitochondrial genomes correspond to diversification rates and to contemporary species richness in birds and reptiles. Proceedings of the Royal Society of London B: Biological Sciences, 277, 3587-3592.

Ericson PG, Christidis L, Cooper A et al. (2002) A Gondwanan origin of passerine birds supported by DNA sequences of the endemic New Zealand wrens. Proceedings of the Royal Society of London B: Biological Sciences, 269, 235-241.

Felsenstein J (1974) The evolutionary advantage of recombination. Genetics, 78, 737-756.

Field, DJ, Lynner C, Brown C and Darroch, SA (2013) Skeletal Correlates for Body Mass Estimation in Modern and Fossil Flying Birds. PLoS One, 8.

Fleischer RC, McIntosh CE, Tarr CL (1998) Evolution on a volcanic conveyor belt: using phylogeographic reconstructions and K-Ar-based ages of the Hawaiian Islands to estimate molecular evolutionary rates. Molecular Ecology, 7, 533-545.

García-Moreno J (2004) Is there a universal mtDNA clock for birds? Journal of Avian Biology, 35, $465-468$.

Page $21 / 26$ 
Garland J Theodore, Ives AR, Travis EJ, Pagel AEM (2000) Using the Past to Predict the Present: Confidence Intervals for Regression Equations in Phylogenetic Comparative Methods. The American Naturalist, 155, 346-364.

Gibb GC, England R, Hartig G et al. (2015) New Zealand Passerines Help Clarify the Diversification of Major Songbird Lineages During the Oligocene. Genome Biology and Evolution, evv196.

Grafen A (1989) The phylogenetic regression. Philosophical Transactions of the Royal Society of London. Series B, Biological Sciences, 326, 119-157.

Grossman LI, Wildman DE, Schmidt TR, Goodman M (2004) Accelerated evolution of the electron transport chain in anthropoid primates. Trends in Genetics, 20, 578-585.

Hackett SJ, Kimball RT, Reddy S et al. (2008) A phylogenomic study of birds reveals their evolutionary history. Science (New York, N.Y.), 320, 1763-1768.

Hall TA (1999) BioEdit: a user-friendly biological sequence alignment editor and analysis program for Windows 95/98/NT. In: Nucleic acids symposium series, pp. 95-98.

Heads M (2005) Dating nodes on molecular phylogenies: a critique of molecular biogeography. Cladistics, 21, 62-78.

Heads M (2011) Old Taxa on Young Islands: A Critique of the Use of Island Age to Date Island-Endemic Clades and Calibrate Phylogenies. Systematic Biology, 60, 204-218.

Ho SYM (2007) Calibrating molecular estimates of substitution rates and divergence times in birds. Journal of Avian Biology, 38, 409-414.

Jarvis ED, Mirarab S, Aberer AJ et al. (2014) Whole-genome analyses resolve early branches in the tree of life of modern birds. Science, 346, 1320-1331.

Jetz W, Thomas GH, Joy JB, Hartmann K, Mooers AO (2012) The global diversity of birds in space and time. Nature, 491, 444-448.

Johnson KP, Seger J (2001) Elevated rates of nonsynonymous substitution in island birds. Molecular biology and evolution, 18, 874-881.

Ksepka D, Clarke JA (2015) Phylogenetically vetted and stratigraphically constrained fossil calibrations within Aves. Palaeontologia Electronica, 18, 1-25.

Page 22 / 26 
Lanfear R, Ho SYW, Love D, Bromham L (2010) Mutation rate is linked to diversification in birds. Proceedings of the National Academy of Sciences, 107, 20423-20428.

Lartillot N, Lepage T, Blanquart S (2009) PhyloBayes 3: a Bayesian software package for phylogenetic reconstruction and molecular dating. Bioinformatics, 25, 2286-2288.

Lehtonen J, Lanfear R (2014) Generation time, life history and the substitution rate of neutral mutations. Biology Letters, 10, 20140801.

Lepage T, Bryant D, Philippe H, Lartillot N (2007) A general comparison of relaxed molecular clock models. Molecular biology and evolution, 24, 2669-2680.

Lerner HRL, Meyer M, James HF, Hofreiter M, Fleischer RC (2011) Multilocus Resolution of Phylogeny and Timescale in the Extant Adaptive Radiation of Hawaiian Honeycreepers. Current Biology, 21, 1838-1844.

Longrich NR, Tokaryk T and Field D J (2011) Mass extinction of birds at the Cretaceous-Paleogene (K-Pg) boundary. Proceedings of the National Academy of Sciences, 108, 15253-15257.

Lott MT, Leipzig JN, Derbeneva O et al. (2013) mtDNA variation and analysis using MITOMAP and MITOMASTER. Current Protocols in Bioinformatics, 1-23.

Lourenço JM, Glémin S, Chiari Y, Galtier N (2013) The determinants of the molecular substitution process in turtles. Journal of evolutionary biology, 26, 38-50.

Lovette IJ (2004) Mitochondrial dating and mixed support for the " $2 \%$ rule" in birds. The Auk, 121, $1-6$.

Manegold A (2007) Earliest fossil record of the Certhioidea (treecreepers and allies) from the early Miocene of Germany. Journal of Ornithology, 149, 223-228.

Mayr G (2009) Paleogene Fossil Birds. Springer.

Mayr G (2013) The age of the crown group of passerine birds and its evolutionary significance-molecular calibrations versus the fossil record. Systematics and Biodiversity, 11, $7-13$

Mayr G, Manegold A (2004) The oldest European fossil songbird from the early Oligocene of Germany. Naturwissenschaften, 91, 173-177.

Page 23 / 26 
Mayr G, Manegold A (2006) A Small suboscine-like Passeriform bird from the early oligocene of France. The Condor, 108, 717.

Mishmar D, Ruiz-Pesini E, Golik P et al. (2003) Natural selection shaped regional mtDNA variation in humans. Proceedings of the National Academy of Sciences of the United States of America, 100, $171-176$.

Mitchell KJ, Cooper A, Phillips MJ (2015) Comment on "Whole-genome analyses resolve early branches in the tree of life of modern birds." Science, 349, 1460-1460.

Nabholz B, Ellegren H, Wolf JBW (2013) High Levels of Gene Expression Explain the Strong Evolutionary Constraint of Mitochondrial Protein-Coding Genes. Molecular Biology and Evolution, 30, 272-284.

Nabholz B, Glémin S, Galtier N (2008) Strong variations of mitochondrial mutation rate across mammals--the longevity hypothesis. Molecular Biology and Evolution, 25, 120-130.

Nabholz B, Glémin S, Galtier N (2009) The erratic mitochondrial clock: variations of mutation rate, not population size, affect mtDNA diversity across birds and mammals. BMC Evolutionary Biology, 9, 54 .

Nguyen JMT, Ho SYW (2016) Mitochondrial rate variation among lineages of passerine birds. Journal of Avian Biology, in press.

Oatley G, Simmons RE, Fuchs J (2015) A molecular phylogeny of the harriers (Circus, Accipitridae) indicate the role of long distance dispersal and migration in diversification. Molecular Phylogenetics and Evolution, 85, 150-160.

Pacheco MA, Battistuzzi FU, Lentino M et al. (2011) Evolution of Modern Birds Revealed by Mitogenomics: Timing the Radiation and Origin of Major Orders. Molecular Biology and Evolution, 28, 1927-1942.

Paradis E (2011) Analysis of Phylogenetics and Evolution with R. Springer.

Pereira SL, Baker AJ (2006) A Mitogenomic Timescale for Birds Detects Variable Phylogenetic Rates of Molecular Evolution and Refutes the Standard Molecular Clock. Molecular Biology and Evolution, 23, 1731-1740.

Page 24 / 26 
Popescu A-A, Huber KT, Paradis E (2012) APE 3.0: New tools for distance-based phylogenetics and evolutionary analysis in R. Bioinformatics, 28, 1536-1537.

Price JP, Clague DA (2002) How old is the Hawaiian biota? Geology and phylogeny suggest recent divergence. Proceedings of the Royal Society of London B: Biological Sciences, 269, 2429-2435.

Prum RO, Berv JS, Dornburg A et al. (2015) A comprehensive phylogeny of birds (Aves) using targeted next-generation DNA sequencing. Nature, 526, 569-573.

Qiu F, Kitchen A, Burleigh JG, Miyamoto MM (2014) Scombroid Fishes Provide Novel Insights into the Trait/Rate Associations of Molecular Evolution. Journal of Molecular Evolution, 1-11.

R Core Team (2013) R: A Language and Environment for Statistical Computing. Vienna, Austria.

Rannala B, Yang Z (2007) Inferring Speciation Times under an Episodic Molecular Clock. Systematic Biology, 56, 453-466.

Reyes A, Gissi C, Pesole G, Saccone C (1998) Asymmetrical directional mutation pressure in the mitochondrial genome of mammals. Molecular Biology and Evolution, 15, 957-966.

Rocha TC, Sequeira F, Aleixo A et al. (2015) Molecular phylogeny and diversification of a widespread Neotropical rainforest bird group: The Buff-throated Woodcreeper complex, Xiphorhynchus guttatus/susurrans (Aves: Dendrocolaptidae). Molecular Phylogenetics and Evolution, 85, $131-140$.

Shen Y-Y, Shi P, Sun Y-B, Zhang Y-P (2009) Relaxation of selective constraints on avian mitochondrial DNA following the degeneration of flight ability. Genome research, 19, 1760-1765.

Shields GF, Wilson AC (1987) Calibration of mitochondrial DNA evolution in geese. Journal of Molecular Evolution, 24, 212-217.

Smith A (2015) Fifteen vetted fossil calibrations for divergence dating of Charadriiformes (Aves, Neognathae). Palaeontologia Electronica, 18.

Smith BT, Klicka J (2010) The profound influence of the Late Pliocene Panamanian uplift on the exchange, diversification, and distribution of New World birds. Ecography, 33, 333-342.

Stamatakis A (2014) RAxML version 8: a tool for phylogenetic analysis and post-analysis of large Page 25 / 26 
phylogenies. Bioinformatics, 30, 1312-1313.

Thomas JA, Welch JJ, Lanfear R, Bromham L (2010) A Generation Time Effect on the Rate of Molecular Evolution in Invertebrates. Molecular Biology and Evolution, 27, 1173-1180.

Thorne JL, Kishino H, Painter IS (1998) Estimating the rate of evolution of the rate of molecular evolution. Molecular Biology and Evolution, 15, 1647-1657.

Trewick SA, Gibb GC (2010) Vicars, tramps and assembly of the New Zealand avifauna: a review of molecular phylogenetic evidence. Ibis, 152, 226-253.

Weir JT, Bermingham E, Schluter D (2009) The Great American Biotic Interchange in birds. Proceedings of the National Academy of Sciences of the United States of America, 106, $21737-21742$.

Weir JT, Schluter D (2008) Calibrating the avian molecular clock. Molecular Ecology, 17, 2321-2328.

Welch J, Bininda-Emonds O, Bromham L (2008) Correlates of substitution rate variation in mammalian protein-coding sequences. BMC Evolutionary Biology, 8, 53.

Woolfit M, Bromham L (2005) Population size and molecular evolution on islands. Proceedings. Biological Sciences / The Royal Society, 272, 2277-2282.

Yang Z (2007) PAML 4: Phylogenetic Analysis by Maximum Likelihood. Molecular Biology and Evolution, 24, 1586-1591.

Yang Z, Rannala B (2006) Bayesian Estimation of Species Divergence Times Under a Molecular Clock Using Multiple Fossil Calibrations with Soft Bounds. Molecular Biology and Evolution, 23, 212-226.

Page 26 / 26 


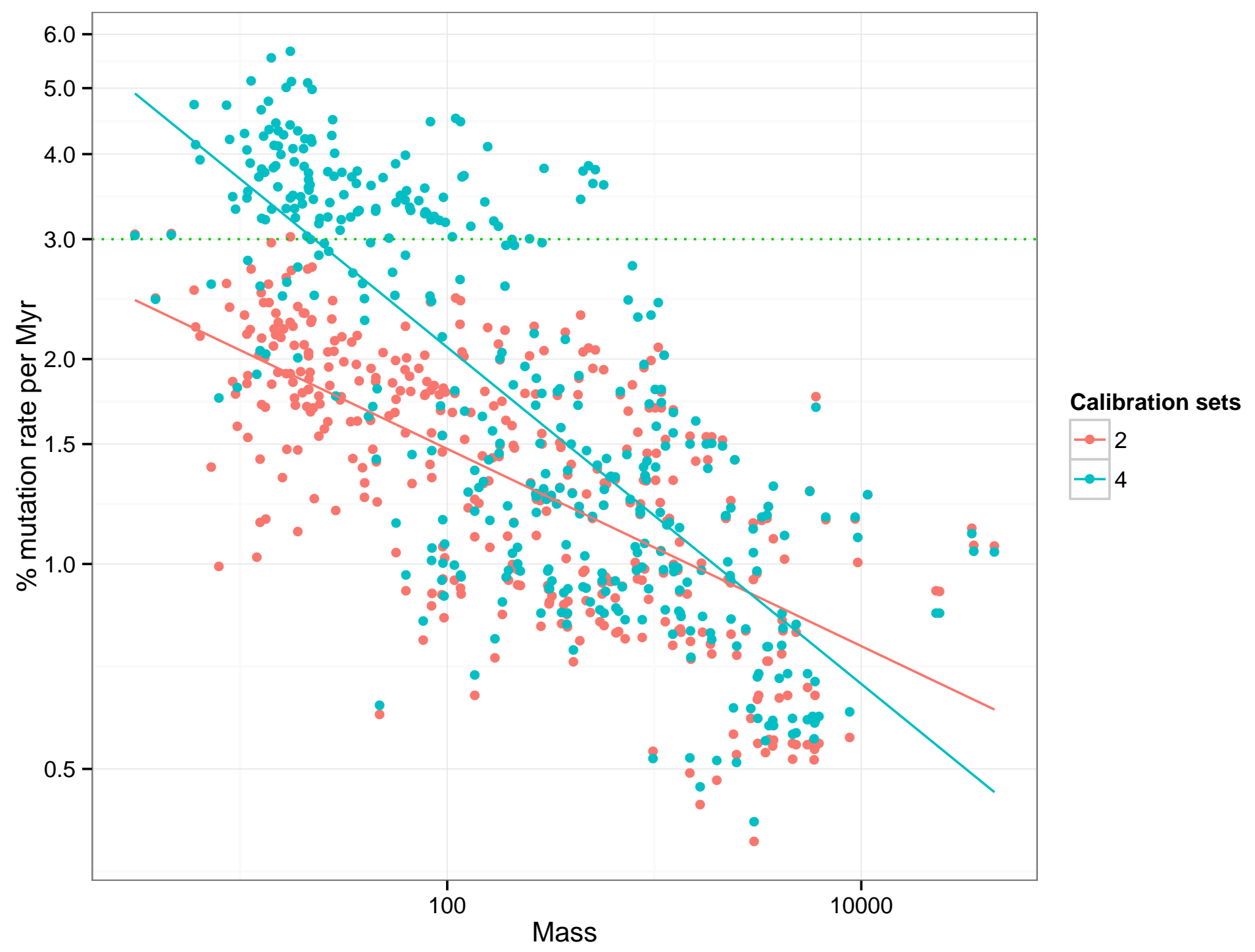


